Revista Posgrado y Sociedad

Sistema de Estudios de Posgrado

Universidad Estatal a Distancia

ISSN $1659-178 X$

Costa Rica

revistaposgradoysoci@uned.ac.cr

Problemática de los ciclos biogeoquímicos, hidrológico y de nutrientes en la meseta central de Costa Rica

Hydrological, nutrients and biogeochemical cycle's disruptions in Costa Rica's central plateau

\author{
Zaidett Barrientos Llosa \\ Universidad Estatal a Distancia \\ San José, Costa Rica
}

Volumen 10, Número 1

Marzo 2010

pp. 23 -37

Recibido: febrero, 2010

Aprobado: marzo, 2010 


\title{
Problemática de los ciclos biogeoquímicos, hidrológico y de nutrientes \\ en la meseta central de Costa Rica
}

\section{Resumen}

La Meseta Central de Costa Rica representa menos del $4 \%$ del territorio del país, pero alberga el $85 \%$ de la industria, el 6o\% de la población, el 70\% de la flota vehicular y los tres acuíferos de mayor importancia. El mayor cambio de uso del suelo se ha dado en los últimos 100 años, y actualmente se pueden reconocer tres zonas básicas: la urbana entre los 800 y los $1400 \mathrm{msm}$, la agrícola y ganadera entre 1300 y $2000 \mathrm{msm}$ y la silvestre entre 1700 y $2800 \mathrm{msm}$. El objetivo de este trabajo es analizar los desequilibrios que ese cambio producto de las actividades humanas han provocado en los ciclos hidrológico, bioquímicos y de nutrientes. La construcción de infraestructura y zonas agrícolas eliminó el bosque, provocando la impermeabilización y la pérdida de suelo fértil. El mal manejo de desechos líquidos y sólidos, y el uso intensivo de agroquímicos, hidrocarburos y detergentes, contamina tanto aguas superficiales como subterráneas, genera un aumento de gases de efecto invernadero, acelera la eutrofización de ríos y lagos, y altera los ciclos biogeoquímicos. Los acuíferos tienen problemas de recarga y sobreexplotación. El suelo presenta condiciones que favorecen la erosión y que impiden la acumulación normal de nutrientes. En conclusión se recomienda tomar medidas para corregir los desequilibrios y mejorar la calidad del ambiente de la región. Entre las medidas correctivas destaca la necesidad de que el gobierno ejerza un verdadero liderazgo; articule mejor las actividades ambientalistas independientes, $\mathrm{y}$ analice y gestione la problemática ambiental dentro de unidades ambientales lógicas. Otras medidas necesarias son: mejorar y asegurar el cumplimiento de las leyes ambientales, mejorar los sistemas de valoración de daños ambientales y promover más investigación y educación ambiental.

Palabras clave: ciclo hidrológico, ciclo biogeoquímico, ciclo de nutrientes, legislación ambiental.

\begin{abstract}
The Central Plateau of Costa Rica represents less than $4 \%$ of the country's territory but hosts $85 \%$ of the industry, $60 \%$ of the population, $70 \%$ of the vehicles and the three most important aquifers. The major land use change has occurred in the past 100 years. Currently three basic areas can be recognized: the urban area between 800 and 1400meters above sea level (masl), the agriculture and livestock area between 1300 and 2000 masl and the wildlife area between 1700 and 2800 masl. Mainly in urban, and agricultural and livestock areas, human activities have caused serious disruptions in the hydrological, nutrients and biogeochemical cycles. The construction of infrastructure and agricultural areas required the removal of the forest, causing the sealing and loss of fertile soil. Mismanagement of solid and liquid waste, and overuse of agricultural chemicals, hydrocarbons and detergents, pollute surface and underground waters, increase greenhouse gases, accelerate lake and river eutrophication and alters biogeochemical cycles. The aquifers have recharge and overuse problems. The soil presents conditions that lead to erosion and prevent normal accumulation of nutrients. A real government leadership is among the measures needed to correct imbalances in the cycles and improve environmental quality. Government leadership should lead to a better articulation between government and independent environmental activities. Environmental problems should be analyzed within a frame of logical environmental units. Other measures that should be taken are: improvement and monitor compliance of environmental laws, improve the valuation of environmental damage and promote more research and environmental education.
\end{abstract}

Keywords: Hydrological cycle, biogeochemical cycles, nutrients cycle, environmental legislation 


\section{Introducción}

Antes del establecimiento de la agricultura y de la excesiva urbanización en la meseta central de Costa Rica, abundaban los bosques y los suelos fértiles. El clima era fresco y agradable a lo largo de todo el año y tenía la fauna propia de un bosque tropical premontano y montano: jilgueros, pavas, jaguares, pumas, saínos, dantas, una gran cantidad de invertebrados y hongos, etc. La mayor parte del territorio permitía la recarga de los acuíferos y los caudales de ríos y manantiales eran relativamente estables a lo largo del año. Los ecosistemas y los ciclos biogeoquímicos al igual que el ciclo hidrológico y el de nutrientes estaban bien balanceados $\mathrm{y}$ gracias a la resilencia podían funcionar dentro de un rango de alteraciones sin que se afecte la globalidad del sistema. No obstante, cuando las alteraciones son muy graves y las condiciones se salen, en uno o mas aspectos, de ese rango en el que trabaja bien la resilencia, el ecosistema colapsa. Es como en un juego de dominó en el que al caer una pieza poco a poco caen las demás. Para lograr mantener un ecosistema gravemente alterado, los humanos utilizamos otros ecosistemas para extraer recursos y depositar desechos, ampliándose los efectos perjudiciales a otros territorios. En este trabajo se analizan los desequilibrios que el cambio de uso de la tierra en la meseta central de Costa Rica está provocando en el ciclo hidrológico, en el ciclo de nutrientes y en los ciclos biogeoquímicos de la región y se plantean algunas medidas que permitirían mejorar las condiciones ambientales actuales de la región.

\section{Cambio de uso del suelo}

La meseta central de Costa Rica es una zona pequeña que mide $1967 \mathrm{~km}^{2}$, lo que equivale al $3.8 \%$ del territorio terrestre del país (MIDEPLAN, 2008). Está delimitada por la cordillera volcánica central al norte y este y los Cerros de Escazú y Ochomogo al sur, su elevación varía entre los 800 msm y los $2900 \mathrm{msm}$ y la precipitación media es de $1967 \mathrm{~mm}$ anuales en las zonas bajas y $3230 \mathrm{~mm}$ en las altas (IMN, 2006). Es importante tener en cuenta que en el sector norte de la meseta están tres de los 12 acuíferos más importantes del país: acuíferos Barba, Colima superior y Colima inferior.

Antes del siglo XVII la región estuvo habitada por poblados muy pequeños de indígenas huetares, pues se calcula que hasta el siglo XVII la población de todo el país nunca pasó de 50 ooo habitantes (Rosero, 2003). A partir del año 1650 la Meseta o Valle Central empezó a ser colonizada por europeos, sin embargo, al inicio, la cantidad de personas que habitaban en esta región aumentó muy lentamente, al igual que la población de huetares. Las principales actividades que realizaban eran la agricultura, ganadería y cacería de subsistencia. En el año de 1820 se importaron las primeras semillas de café; los buenos resultados que dio ese cultivo motivaron a que se ampliaran las áreas de siembra pues se buscaba la exportación del producto. Este hecho y 
las mejoras en el sistema de salud nacional permitieron que la población aumentara a 250 mil habitantes en 1892, y se incrementara a un millón en 1956 (Rosero, 2003). Sin duda, el mayor aumento poblacional se ha dado en los últimos 100 años pues para el año 2009 se contaba con 4.5 millones (INEC, 2009).

La Meseta Central es la región de Costa Rica que se ha visto mas severamente afectada por ese aumento poblacional y por el cambio en las actividades económicas del país y el consecuente cambio en el uso del suelo. Además, con el transcurso del tiempo, se ha presentado el fenómeno de conurbación, lo que significa que los pueblos al ir aumentando de tamaño se unen por una extensa red vial y de caseríos. Desafortunadamente, este proceso se da sin una buena planificación lo que provoca que los sistemas administrativos y la infraestructura colapsen al igual que el ecosistema. Para la primera década del siglo XXI, a pesar de la poca extensión territorial, la meseta central alberga al $85 \%$ de las industrias del país (Valdéz, 2002), a las cuatro ciudades mas importantes (San José, Cartago, Alajuela y Heredia) y al 60\% de la población de Costa Rica (Alfaro, 2001).

Actualmente el uso de la tierra en el valle central se sectoriza en tres: a) zona urbana e industrial, esta zona forma una capa casi uniforme entre los $800 \mathrm{msm}$ y los $1400 \mathrm{msm}$ aproximadamente, todavía quedan algunos potreros y plantaciones de café, pero el alto valor de la tierra favorece que esas áreas se transformen en residenciales y zonas industriales; b) zona agrícola y ganadera, ubicada entre los 1300 msm y los 2000 msm, esta zona se está perdiendo y está dando paso a residencias tipo quinta con amplios espacios verdes, que por lo general son despojados de su cobertura boscosa natural; y c) zona de áreas silvestre, después de los 1700 ó 2000 msm se ubican las zonas protegidas y los remanentes de bosque que llegan hasta los 2800msm.

\section{Problemas que amenazan la estabilidad del ecosistema}

Como resultado del cambio de uso del suelo y de la concentración de humanos y de sus actividades se tienen varios problemas que han llevado al ecosistema de la región fuera de su rango de resilencia, alterando el ciclo del agua, el ciclo de nutrientes y los ciclos biogeoquímicos. Los principales problemas y consecuencias que podemos mencionar son: deforestación, erosión y eliminación de la capa fértil del suelo, impermeabilización del suelo, inundaciones, contaminación de aguas superficiales y subterráneas, acumulación de desechos sólidos, cambio en los patrones de temperatura, problemas de recarga de los acuíferos, sobre explotación del recurso hídrico y mal aprovechamiento de los suelos. A continuación se hablará sobre algunos de estos problemas, pero, aunque se traten en forma separada, se debe tenerse en cuenta que se interrelacionan.

\section{Alteración en el ciclo hidrológico}

El ciclo hidrológico, también conocido como ciclo del agua, permite el reciclaje natural del agua, manteniéndola limpia y en cantidad suficiente para satisfacer nuestras necesidades. Las principales fuentes de abastecimiento de agua de la Meseta Central son: agua superficial 
proveniente de otras regiones y un vasto sistema de pozos legales e ilegales que extraen agua de los mantos acuíferos de la región (en este trabajo solo se analiza la meseta central, pero debe quedar claro que al tomar agua de otros lugares o al trasladar desechos estamos afectando otros ecosistemas). Los principales problemas que presenta el recurso hídrico son la sobreexplotación, la contaminación y la impermeabilización.

\section{- Sobreexplotación}

Las aguas superficiales de la región se aprovechan relativamente poco, pues solo en las zonas altas tienen la calidad suficiente para ser desviadas para uso humano. En general hay poco control de la cantidad de agua que es aprovechada de esta forma ya que su consumo es administrado por organizaciones comunales encargadas del abastecimiento de agua (ASADAS) que no llevan un buen control del volumen utilizado. Aunque en la Meseta Central hay cinco acuíferos libres y superficiales (Barba, Poas, La Libertad, Los Angeles y Los Bambinos) y dos acuíferos confinados (Colima Superior y Colima Inferior) el aprovechamiento de aguas subterráneas se centra en los tres acuíferos mas grandes, los cuales se encuentran sobrepuestos: Barba, el mas superficial, con cerca de $135 \mathrm{~km}^{2}$, Colima Superior con una extensión aproximada de $230 \mathrm{~km}^{2}$ y Colima Inferior, el que se encuentra a mayor profundidad, con aproximadamente 130 km² (OPS, 2003; Municipalidad de San Rafael, 2008; Arias, 2006). Estos tres acuíferos probablemente brindan más del 50\% del agua que se utiliza en la meseta. El total estimado de agua que se aprovecha en pozos y manantiales varía entre $2800 \mathrm{l} / \mathrm{s}$ y $9870 \mathrm{l} / \mathrm{s}$, la variación en los datos se deben principalmente a que el acuífero Barba tiene una gran cantidad de pozos ilegales, y a que además, no hay un buen control del volumen que efectivamente se extrae en los pozos legales (Fernández González et al., 2001; Ramírez, 2007).

En total se ha calculado que el sistema de acuíferos de la meseta central recibe una recarga promedio de entre 8200 y $9720 \mathrm{l} / \mathrm{s}$, sin embargo, estos cálculos no consideran el efecto de la impermeabilización del terreno por lo que el monto real de recarga puede ser menor (Arias, 2006; Ramírez, 2007). En los puntos de mayor explotación se registran descensos del nivel freático de hasta 20m y reducciones en el caudal de algunos manantiales (Arias, 2006). Por lo tanto todo parece indicar que el recurso está siendo sobre explotando.

\section{- Contaminación}

El mal manejo de desechos sólidos y líquidos ha provocado que los ríos se llenen de toda clase de contaminantes. Muchas de las industrias y residencias que se ubican cerca de cauces de ríos vierten sus efluentes sin tratamientos previos, pese a que existe la ley de vertidos que impide esta práctica. De hecho en una encuesta realizada por el Laboratorio de Ecología Urbana de la UNED (en elaboración) a los vecinos de ríos urbanos, mencionaron como una ventaja de vivir a la par del río, el hecho de no tener que construir tanques sépticos ya que pueden verter las aguas servidas directamente al río. La gran 
debilidad de esta ley radica en la falta de controles $y$, dado el costo del establecimiento y mantenimiento de plantas de tratamiento, la ley se infringe con mucha frecuencia. Por otra parte, el sistema de recolección de desechos sólidos se encarga de desechos de tamaño relativamente pequeño y no recolecta desechos como llantas, muebles, refrigeradores, etc. Ante el alto costo de llevarlos a un relleno sanitario las personas los botan en los ríos, lo mismo hacen con el resto de los desechos cuando falla el sistema de recolección municipal.

Los contaminantes vertidos en los ríos elevan la demanda biológica $\mathrm{y}$ química de oxígeno y consecuentemente disminuye el oxígeno disuelto en el agua, provocando la muerte de la flora $y$ fauna $y$ aumentando las poblaciones de bacterias anaeróbicas. Se tiene entonces un río mal oliente, de aguas estancadas en donde proliferar plagas de zancudos y de ratas que se alimentan de los desechos acumulados. La mala impresión que da la contaminación de los ríos afecta negativamente al turismo que es la principal entrada de divisas del país y causa cuantiosos gastos de limpieza y drenaje en la planta hidroeléctrica Brasil ubicada en el río Virilla en donde se extraen 914 toneladas de basura por año (Loaiza, 2007). Además se disminuye la calidad del ambiente en el que viven los vecinos de estos ríos.

$\mathrm{Al}$ igual que las aguas superficiales, los acuíferos están expuestos a la contaminación, pero tienen el agravante de que su restauración es mas lenta y costosa, ya que los procesos naturales requieren de tanto tiempo que no pueden ser considerados como viables (Reynolds,
1996). Esta zona posee suelos andisoles muy permeables por lo que el derrame de cualquier sustancia sobre el suelo representa un peligro potencial de contaminación para los acuíferos libres. El acuífero mas vulnerable es el Barba por ser libre y el mas superficial, es decir que carece de una capa impermeable que lo proteja y que el nivel freático se encuentra a poca profundidad. Ramírez y Alfaro (2002) clasificaron toda la zona norte y oeste del Valle Central (norte de la ciudad de Heredia hasta Alajuela en el oeste y San Isidro de Heredia en el este) al igual que el norte y este de San Isidro de Coronado como de alta vulnerabilidad debido a que el nivel freático esta a menos de 30 metros de profundidad. Según estos autores los lugares mas poblados de la meseta central se encuentran ubicados en una zona de vulnerabilidad media, y recomiendan que la urbanización de esas zonas no exceda 250 personas por hectárea y que haya sistemas de alcantarillado y de tratamiento de aguas. En cuanto a los acuíferos Colima Superior y Colima Inferior que están subpuestos al Barba $\mathrm{y}$ que son confinados (tienen capas impermeables en gran parte de su extensión), se calcula que el área de alta vulnerabilidad a la contaminación es apenas del $2 \%$ y se ubica cerca del cauce del río Virilla que es donde estos acuíferos son mas superficiales y están mas cercanos a las actividades urbanas (OPS, 2003; Agüero, 2000; Reynolds, 1996; Municipalidad de San Rafael, 2008; Arias, 2006; UNEP, 2006). Sin embargo, hay dos aspectos que no se consideraron en ese estudio y que elevarían en mucho la vulnerabilidad de estos acuíferos: uno es la posibilidad de que en algún punto, o época del año, el río Virilla alimente a los acuíferos, lo 
cual podría perjudicar la calidad del agua de estos acuíferos dada la alta contaminación de este río (Agüero, 2000) y el otro aspecto es que la contaminación del acuífero Barba podría afectarlos si existen fracturas en las capas impermeables que los separan y que permitirían que el acuífero Barba alimente a los otros dos por percolación vertical (OPS, 2003; Agüero, 2000; Reynolds, 1996; Municipalidad de San Rafael, 2008; Arias, 2006; UNEP, 2006; Ramírez, 2007).

No todo lo que derramamos sobre el suelo llega hasta los acuíferos. Afortunadamente los contaminantes que se derraman sobre el suelo sufren procesos atenuantes: la actividad microbiológica del suelo puede degradar muchos compuestos orgánicos, las raíces pueden absorber algunas sustancias, el suelo también tiene la capacidad de adsorber sustancias y aunque el suelo es muy permeable, la infiltración es lenta ( 2 a 3,5 metros por año). No obstante, con el tiempo y el exceso de contaminantes, algunas sustancias ya han logrado llegar al acuífero Barba (Reynolds et al., 1994, 1995). Aunque no grave, se ha demostrado que existe contaminación por nitratos y por compuestos orgánicos volátiles (Reynolds, 2005). La principal fuente de este problema es el uso generalizado de tanques sépticos, seguido por el mal manejo de fertilizantes nitrogenados en el cultivo del café. Como ya se mencionó, las plantaciones de café están siendo sustituidas por urbanizaciones, por lo que se debe prestar especial atención a este sistema de tratamiento de aguas servidas domiciliares, ya que entre el 2000 y el 2005 su uso aumentó del 70 al 75\% de la población (Campus, 2004; Agüero, 2010). Desafortunadamente, actualmente no existe una normativa que obligue y vigile por que se de el mantenimiento que estos sistemas requieren.

Aparte, de la contaminación por los tanques sépticos, el incidente mas dramático en la contaminación de los acuíferos de la región fue el derrame en setiembre de 2004 de mas de 20000 litros de gasolina por una fuga en los tanques de almacenamiento de una gasolinera en la zona franca de Belén (Fallas, 2007), a finales del 2009 la gasolinera seguía clausurada, pero no se han puesto en práctica medidas de restauración por parte de la empresa responsable (Solano \& Bravo, 2007).

\section{- Recarga e impermeabilización}

La impermeabilización del suelo tiene tres consecuencias dentro del ciclo hidrológico: primera el agua no logra infiltrarse y abastecer los mantos acuíferos, segunda el volumen de escorrentía aumenta provocando erosión tanto del terreno como de las paredes de los cauces, provocando sedimentación de los ríos e inundaciones (Solano et al., 2002) y tercera durante la época seca, el caudal de los ríos disminuye ya que el acuífero no se ha abastecido adecuadamente durante la época de lluvias, provocando un mal flujo, estancamiento de agua y acumulación de desechos acarreados por el exceso de escorrentía y de origen humano.

La razón por la cual se ha impermeabilizado el suelo en la Meseta Central es el cambio de uso del suelo. Los terrenos que antes estaban cubiertos principalmente por bosques y 
cafetales con sombra, ahora, en su mayoría, son parte de la red vial, residencias, industrias, comercio y zonas verdes no silvestres (Solano et al., 2002). Actualmente, la red vial de la Meseta Central es insuficiente $y$ requiere de ampliaciones, modernizaciones y diseño de toda una estrategia para enfrentar las aglomeraciones que producen contaminación atmosférica, por lo que es probable que en el futuro aumente el área impermeabilizada por esta razón (Brenes, 2009; Flores, 2009).

Las zonas verdes no silvestres, o con manejo humano, también pueden ser consideradas como parte del problema de impermeabilización del suelo y de mal manejo del recurso hídrico porque a diferencia de las zonas verdes silvestres: tienen grandes extensiones enzacatadas, $\mathrm{o}$ incluso asfaltadas, se ha plantado una gran cantidad de vegetación exótica y poco adaptada al clima de la región, poseen poca densidad de árboles y carecen de la hojarasca que normalmente cubre los bosques. El zacate, al igual que la mayor parte de las plantas ornamentales, requiere de mucha agua en verano para mantenerse verde, contribuyendo a la sobreexplotación del recurso. Además, esa vegetación de escasa altura y poca densidad no logra mantener una temperatura estable en los primeros centímetros del suelo, como si lo hace el bosque, por lo tanto el agua de lluvia se evapora más rápidamente y disminuye la cantidad de agua que se infiltra hacia los acuíferos. Por otra parte, en las zonas verdes que tienen manejo humano se elimina toda la hojarasca por considerarse fea y se tiene poca conciencia del papel que esta cumple en el ciclo del agua. Cuando llueve, el suelo se satura rápidamente y da inicio el proceso de infiltración que es bastante lento. El suelo no puede absorber mas agua hasta que parte del agua que retuvo se evapore, sea absorbida por la vegetación o se infiltre, entonces el agua extra que cae corre por escorrentía por la superficie del suelo hasta el arroyo más cercano. Sin embargo, si hay una buena capa de hojarasca, esta acumula parte de esa agua "extra" y protege la humedad del suelo de la evaporación, dándole más tiempo al proceso de infiltración. Una buena cobertura boscosa, además de ayudar a mantener una temperatura estable (FAO, s.f.), permite prolongar el tiempo de infiltración gracias al goteo, el cual se puede prolongar hasta por 30 minutos después de llover. Tanto el bosque como la hojarasca, protegen al suelo, también, de la acción desecante del viento. Así, pues aunque en las zonas verdes urbanas si hay infiltración, esta es parcial, y dista mucho de igualar el aporte que da un bosque natural al ciclo hidrológico.

Como ya se mencionó la recarga de los acuíferos del Valle Central se da por infiltración de la lluvia y por percolación vertical de un acuífero a otro. La impermeabilización afecta principalmente al acuífero Barba, que es el más superficial de la región (Reynolds, 1996) y que se alimenta por infiltración en toda su extensión, la cual coincide con las zonas medias y bajas de la Meseta Central que son las áreas que han sufrido un mayor cambio de uso de la tierra. Los acuíferos Colima Superior y Colima Inferior tienen sus principales zonas de recarga en la zona media y alta del Valle Central, principalmente en los cantones de Alajuela, Santo Domingo, San Rafael, San Isidro, Santa Barbara, Barva, Goicoechea, Moravia y Vásquez de 
Coronado (Ramírez, 2007). En la zona media aunque hay un poco mas de cobertura vegetal que las zonas bajas, pero esta no es la cobertura natural, pues se trata principalmente de pastizales y sembradíos, que para efectos del ciclo hidrológico sufren de los mismos problemas que se mencionaron anteriormente para las zonas verdes urbanas. Por lo tanto, en estas zonas de alturas intermedias se produce una mayor escorrentía y una tasa de evaporación mayor del agua contenida en la tierra, que la que hay en un bosque. Las zonas altas de la región si están protegidas y por lo que la principal zona de recarga de los acuíferos Colima Superior y Colima Inferior solo se ven afectadas por el constante avance de la urbanización.

Una de las consecuencias de la impermeabilización, o incapacidad del suelo de absorber agua, es el aumento de inundaciones. En la meseta central existe un registro grande de este tipo de desastres y se ha encontrado que su número e impacto ha aumentado con el tiempo (Solano et al., 2002), pero también hay que tener en cuenta que con el aumento poblacional, se han construido viviendas en lugares de mayor riesgo de lo que se hacía antes por lo que los efectos de las inundaciones son mas tangibles ahora (Arroyo, 2009). Recientemente las inundaciones más grandes y de mayor impacto han sido provocadas por la quebrada Seca en el cantón de Belén (Sánchez, 2007; Municipalidad de Belén, 2008). Como respuesta a los problemas de inundación, contaminación, malos olores, mala planificación, etc. se han entubado gran cantidad de cauces en la zona metropolitana. Esta opción además de irrespetar la Ley Forestal 7575 artículo 33, es claramente una forma de ampliar el terreno para construcciones, la cual probablemente es la verdadera razón de esa medida, pues el entubamiento no arregla el origen del problema (Sagot, 2007).

\section{- Legislación}

En Costa Rica existe una extensa lista de instituciones que se encargan de asuntos relacionados con el agua (Acueductos y Alcantarillados, Instituto Costarricense de Electricidad, Empresa de Servicios Públicos de Heredia, municipalidades, entre otros) y de leyes, decretos y reglamentos que buscan la protección del recurso hídrico (Ley Orgánica del Ambiente, Ley de la Conservación de la Vida Silvestre, Reglamento de Creación de Canon Ambiental por Vertidos, Reglamento de Vertido y Reuso de Aguas Residuales, entre otros), aunque todavía hay cabos sueltos e inconsistencias en la legislación, la falta de regulaciones no es tan grave como: 1) la falta de cumplimiento de las mismas y 2) la falta de articulación de las leyes dentro del concepto de gestión integral de cuencas.

La falta de cumplimiento de las leyes es producto de que el país no ha destinado los recursos suficientes para crear mecanismos eficientes para vigilar el cumplimiento de la legislación, sancionar a los infractores y asegurarse de que los daños ambientales sean correctamente evaluados y valorados y las medidas de restauración sean cumplidas. Un ejemplo es el caso de la contaminación de aguas superficiales por la 
cooperativa de leche Dos Pinos: el castigo que se impuso fue mínimo comparado con el daño causado (Ruiz, 2009).

El segundo aspecto hace que muchos de los esfuerzos por proteger el recurso hídrico no estén bien encauzados porque los problemas se ven de forma aislada, cuando en realidad se trata de un sistema complejo en el cual la suma de las partes no es igual a la totalidad de la problemática. Un buen paso en la búsqueda de soluciones de manera integral es el inicio en el 2010 de la construcción de una única planta de tratamiento de las aguas servidas de gran parte de la Meseta Central, la cual se espera que esté lista para el 2012 y tenga una vida útil de 30 años (Avalos, 2009). Este será un gran aporte, pues así se estaría tratando al $75 \%$ de las aguas servidas de la región (Agüero, 2010).

\section{Alteraciones en el ciclo de nutrientes}

Debido a la actividad pasada de los volcanes que rodean la Meseta Central, el suelo de esta región es muy fértil, pero el constante crecimiento demográfico le ha dado gran plusvalía a los terrenos agrícolas y se ha promovido el cambio de uso, favoreciendo la urbanización. La construcción de infraestructura, residencias, comercio, industrias $\mathrm{y}$ zonas verdes urbanas requiere la eliminación del bosque y del detrito que cubre el suelo, por lo que el país ha perdido sus mejores suelos agrícolas.

En el caso del terreno destinado a la agricultura aunque se elimina el bosque casi totalmente, no se elimina el detrito, sin embargo, la destrucción o eliminación de los rastrojos, la recolección de cosechas y la ausencia de la hojarasca producida por el bosque impiden que se mantenga el ciclo de nutrientes por lo que el suelo se ve cada vez mas empobrecido y requiere de fertilizantes y abonos para continuar produciendo. Como ya se mencionó, el uso excesivo de fertilizantes provoca contaminación de las fuentes de agua con nitrógeno, uno de los principales componentes de los fertilizantes, el cual es acarreado hasta los mantos acuíferos por la lluvia que se infiltra (Reynolds et al., 1994, 1995; OPS, 2003). La agricultura y la ganadería contribuyen con la contaminación de las fuentes de agua por amplio uso de plaguicidas.

\section{Alteración de los ciclos biogeoquímicos}

El ciclo del carbono se altera principalmente al quemar hidrocarburos y al manejar mal nuestros desechos sólidos y líquidos, ya que se libera $\mathrm{CO}_{2}$ y $\mathrm{CH}_{4}$ (metano) a la atmósfera. Ambos gases tienen efecto invernadero, entonces al aumentar su cantidad en la atmósfera, aumenta la temperatura del planeta. Un ambiente mas caliente favorece la evaporación y la evapotranspiración, aumentando la concentración de vapor de agua, otro gas con efecto invernadero, estableciéndose un ciclo vicioso de desequilibrios. En comparación con otros países el aporte que hace la meseta central al calentamiento global es muy pequeño, pero es un aporte que debemos manejar adecuadamente.

No hay datos exactos del consumo de hidrocarburos en la meseta central, pero se puede extrapolar su importancia si consideramos que en esta zona se está el 70\% de la flota vehicular del país (Alfaro, 2001) y que el transporte terrestre es la principal 
fuente de consumo de petróleo en Costa Rica el cual aumenta en un $8 \%$ anual (IndexMundi, 2008). Si se quieren establecer lineamientos para disminuir el consumo de hidrocarburos se debe considerar que: la cantidad de vehículos aumenta constantemente independientemente de los cambios en el precio del petróleo con un aumento anual promedio del 10\% (Chacón, 2000); hay uso ineficiente del transporte ya que muchos camiones viajan vacíos y muchos vehículos llevan solo un tripulante (Chacón, 2000); las rutas no son óptimas por lo que el congestionamiento, el mal estado de las calles, las rutas obsoletas e innecesariamente fragmentadas provocan gasto innecesario de combustible; y no hay una política que fomente el uso de transporte alternativo y tecnología moderna y no contaminante. Los desechos sólidos y líquidos también generan $\mathrm{CO}_{2}$ y $\mathrm{CH}_{4}$. En la meseta central hay 5 rellenos sanitarios legales pero también hay varios botaderos que reciben aproximadamente 2200 toneladas de desechos por día (Programa Cyma, 2008).

El consumo de combustibles fósiles y los procesos de descomposición también hacen que aumente la concentración de azufre en el aire el cual reacciona con el vapor de agua y produce lluvia ácida. La lluvia ácida puede matar las plantas provocando un efecto similar a la deforestación, con el agravante de que daña la fertilidad del suelo. Además, en las ciudades produce daño a las construcciones de cemento y metal. En la meseta central ya se han dado registros de lluvia ácida que deterioran la infraestructura patrimonial por ejemplo el Teatro Nacional (Villegas, 2005). Un estudio del 2005 detectó un $\mathrm{pH}$ ligeramente ácido, aunque los datos nunca excedieron de $\mathrm{pH} 5$ (Villegas, 2005), por lo que no es un problema grave.

El ciclo del nitrógeno también es afectado por las actividades humanas. Los organismos liberan nitrógeno en sus excretas y al morir, y son las bacterias descomponedoras las que se encargan de reincorporarlo al suelo para que sea aprovechado por las plantas. Sin embargo, cuando la cantidad de materia en descomposición o de excretas es mucha, el nitrógeno es acarreado por el agua hasta los acuíferos, contaminándolos. Considerando que en la región se ha eliminado la mayor parte del suelo fértil en donde se encuentran las bacterias descomponedoras y al incremento en el uso de tanques sépticos es imprescindible tomar medidas para el manejo correcto de las aguas negras (OPS, 2003; Agüero, 2010).

Los humanos extraemos el fósforo de sus depósitos naturales para hacer fertilizantes y detergentes. Pero luego los residuos, cargados de fósforo, son trasladados por la lluvia y las aguas servidas sin tratamiento hasta los ríos y lagos, en donde funcionan como fertilizante para las plantas acuáticas y algas, favoreciendo la eutrofización. En el río Virilla se ha encontrado que el límite de concentración de fosfatos permitido se sobrepasa hasta en 3468 veces (máximo permitido $2.5 \mathrm{mg} / \mathrm{L}$, máximo encontrado $8670.0 \mathrm{mg} / \mathrm{L}$ ) (Fernández et al., 2001). 


\section{Conclusión}

La problemática de la alteración de los ciclos hidrológico, de nutrientes y biogeoquímicos en Costa Rica es muy compleja y su solución debe verse de manera integral en unidades ambientales lógicas. Por esa razón el enfoque de gestión integrado de cuencas es una buena opción de solución, pues se incluyen los diferentes aspectos sociales, económicos y ambientales que afectan a una unidad ambiental. Costa Rica cuenta con 34 cuencas y sus elementos ambientales y sociológicos son únicos, por lo que es necesario hacer un análisis individual de cada una. La Meseta Central se ubica en la subcuenca del Virilla dentro de la cuenca del río Tárcoles y tiene como principales características que es la región más poblada e industrializada del país y que posee los tres acuíferos mas importantes de Costa Rica.

Los esfuerzos en investigación, extensión, educación y gestión desarrollados por Universidades (p.e. análisis de características hidrológicas de la región), por las municipalidades y otras entidades gubernamentales (p.e. campañas de recolección de desechos, de siembra de árboles como "a que sembrás un árbol"),organizaciones comunales (por ejemplo Bandera Azul ecológica) y por organizaciones privadas (por ejemplo Bioland S.A. que tiene un programa de reforestación) no tienen una buena articulación entre si y no están bien respaldados por una legislación coherente que visualice los problemas ambientales desde una perspectiva de una unidad ambiental lógica, por lo que los resultados obtenidos se ven diluidos en la realidad regional. Aunque obviamente se deben aprovechar todas estas iniciativas, el gobierno debe establecer mejor su liderazgo y el rumbo que se debe seguir para la protección de los recursos de la región. El desarrollo que ha tenido la meseta central ha producido desequilibrios importantes en los ciclos analizados en este trabajo y es importante establecer medidas que permitan detener el avance de la degradación y en el mejor de los casos sanar esos desequilibrios. A continuación se enlistan algunas posibles medidas:

Como ha mencionado Dengo (2007) es decisivo el papel de liderazgo del gobierno tanto para hacer cumplir las leyes como para establecer un buen ordenamiento territorial y organizar el trabajo conjunto de los diferentes actores sociales en pro de un ambiente que garantice una buena calidad de vida para todos los habitantes:

1.Fomentar la investigación que permita guiar técnicamente la restauración de los ciclos y ecosistemas afectados por las actividades humanas.

2. Determinar con mayor exactitud cual es el área de recarga de los acuíferos Colima Superior y Colima Inferior, en caso de ser necesario se debería ampliar la cobertura del Parque Nacional Braulio Carrillo para la correcta protección de las principales zonas de recarga o bien fortalecer mecanismos alternos como el pago de servicios ambientales.

3. Medir con exactitud el consumo de aguas subterráneas, teniendo un mejor control de la cantidad real extraída en cada pozo y documentando los pozos ilegales.

4. Fomentar la protección del ambiente mediante legislación 
adecuada y medidas dictadas por el gobierno, por ejemplo: establecer técnicas de producción mas limpia o de gestión ambiental en todas las actividades humanas, agricultura orgánica en zonas de recarga, regular el uso de detergentes fosfatados y de sistemas de tratamiento de aguas servidas.

5. Establecer sistemas de retroalimentación ágil que permita modificar con prontitud la legislación y los mecanismos de fiscalización y evaluación de daño ambiental.
6. Continuar y mejorar los programas de educación ambiental de toda la población.

7. Mejorar la planificación urbana, de manera que las nuevas obras que se realicen consideren la existencia de zonas verdes silvestres y manejadas que permitan satisfacer las necesidades sociales y ambientales de la región, que sirvan como corredores biológicos, zonas de recarga de los acuíferos y protección del suelo y que a la vez sirvan de áreas de esparcimiento y sean seguras.

\section{Referencias}

Agüero, J. (2000). Análisis de vulnerabilidad a la contaminación de una sección de los acuíferos del Valle Central de Costa Rica. Proyecto de graduación. Escuela de Ingeniería Civil. Recuperado el 16 de setiembre del 2009 en http://www.produs.ucr.ac.cr/tesis/Jonathan\%20Ag\%FCero.pdf

Agüero, M. (2010). País perdió en alcantarillado sanitario. La Nación 10 enero 2010. Recuperado el 11 de enero del 2010 en http://www.nacion.com/ln_ee/2010/enero/10/pais2143146.html

Alfaro, R. (2001). Contaminación del aire en la capital. Ambientico 93. Recuperado el 16 de setiembre del 2009 en www.una.ac.cr/ambi/Ambien-Tico/93/alfaro.htm

ANEP. S.f. Ingenio Taboga cuestionado por daño ambiental. Recuperado el 20 de octubre del 2009 en http://www.anep.or.cr/leer.php/1077

Arias, M., Losilla, M. y Arredondo, S. (2006). Estado del conocimiento del agua subterránea en Costa Rica. Boletín geológico y minero 117 (1): 63-73.

Arroyo, L.N. (2009). Inundaciones en Costa Rica: esbozo tipológico y estrategias de mitigación. Ambientico 191: 3-4. Recuperado el 26 de noviembre del 2009 en http://www.ambientico.una.ac.cr/191.pdf

Avalos, A. (2009). AyA busca fondos para tratar aguas negras. La Nación 26 de marzo de 2009.

Brenes, E. (2009). Plan Prugram 2008-2030: Propuesta de viabilidad y transporte en la Gran Area Metropolitana. Ambientico 188: 3-6. Recuperado el 30 de noviembre del 2009 en http://www.ambientico.una.ac.cr/188.pdf

Campus. (2004). Situación del agua en Costa Rica. Resumen ejecutivo. Recuperado el 25 de noviembre del 2009 en http://www.una.ac.cr/campus/ediciones/otros/agua.pdf

Chacón, R. (2000). Uso de hidrocarburos en transporte y contaminación en Costa Rica. Ambientico 81. Recuperado el 11 de octubre del 2009 en http://www.una.ac.cr/ambi/revista/81/Chacon.htm

Dengo, J.M. (2007). Perspectivas y problemática en el uso del agua. Biocenosis 20 (1-2): 5-14.

Fallas, H. (2007). Agua potable en riesgo de contaminación química. La Nación 12 abril 2007. Recuperado el 04 de octubre del 2009 en http://www.nacion.com/ln ee/2007/abril/12/pais1055676.html

FAO. S.f. Introduction to evapotranspiration. Recuperado el 25 de noviembre del 2009 en http://www.fao.org/docrep/x0490E/x0490e04.htm

Fernandez-Gonzalez, A., Blanco, V. y Gutiérrez-Espeleta, E. (2001). El agua en Costa Rica: abundante pero vulnerable. Boletin del observatorio del desarrollo 3 (6): 1-8. Recuperado el 29 de noviembre del 2009 en http://www.odd.ucr.ac.cr

Flores, R. (2009). Estancamiento de infraestructura y transportes en la Gran area Metropolitana. Ambientico 188: 7-9. Recuperado el 25 de noviembre del 2009 en http://www.ambientico.una.ac.cr/188.pdf 


\section{Problemática de los ciclos biogeoquímicos, hidrológico y de nutrientes}

en la meseta central de Costa Rica

IMN. (2006). Clima en Costa Rica: Valle central. Recuperado el 27 de noviembre del 2009 en http://www.imn.ac.cr/educacion/climacr/index.html

INEC. (2009). Instituto Nacional de Estadística y Censo. Recuperado el 04 de octubre del 2009 en http://www.inec.go.cr/

IndexMundi. (2008). Costa Rica: Petróleo y consumo. Recuperado el 11 de octubre del 2009 http://www.indexmundi.com/es/costa rica/petroleo consumo.html

Loaiza, V, (2007). CNFL pierde ф1.60o millones al año por basura en embalses. La Nación, versión

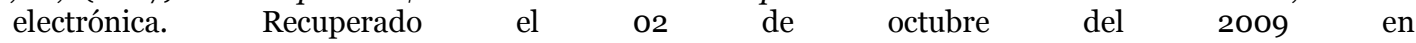
http://www.nacion.com/ln_ee/2007/agosto/12/pais1199722.html

MIDEPLAN. (2008). Política de sostenibilidad ambiental. Boletín del ministerio de planificación nacional y política económica 2 (3): 1-20. Recuperado el 04 de octubre del 2009 en http://www.mideplan.go.cr/component/option,com docman/task,doc view/gid,144/

Municipalidad de Belén. (2008). Comité local de emergencia del cantón de Belén. Recuperado el o2 de octubre del 2009 en http://www.belen.go.cr:800o/comision_emergencias.jsp

Municipalidad de San Rafael. (2008). Estudios generales: Hidrogeología. Recuperado el 18 de setiembre del 2009 en http://www.munisrh.go.cr/estudios generales.htm

OPS. (2003). Calidad del agua potable en Costa Rica: Situación actual y perspectivas. Organización Panamericana de la Salud- Ministerio de Salud. 4op.

PROGRAMA ESTADO DE LA NACION. 2008. Informe 14: Armonía con la naturaleza. Recuperado el o3 de octubre del 2009 en http://www.estadonacion.or.cr/Info2008/Paginas/armonia.html\#Recursos

Programa Cyma. 2008. Plan de Residuos Sólidos Costa Rica. Recuperado el 24 de octubre del 2009 en http://www.progai.ucr.ac.cr/documentos/conferencias magistrales/Plan\%20de\%20Residuos\%20Solid os\%20Costa\%20Rica.pdf

Ramírez, R. (2007). Recarga potencial del acuífero Colima y Barba, Valle central, Costa Rica. Servicio nacional de aguas subterráneas, riego y avenamiento. Recuperado el o5 de octubre del 2009 en http://endefensadelagua.org/index.php?option=com docman\&task=doc details\&gid=2\&Itemid $=48$

Ramírez, R. \& A. Alfaro. (2002). Mapa de vulnerabilidad hidrogeológica de una parte del valle central de Costa Rica. Revista geológica de América Central 27: 53-60. Recuperado el 28 de enero del 2010 http://www.geologia.ucr.ac.cr/revista/to_pdf/revista/27/27-RAMIREZ.pdf

Reynolds-Vargas, J. (1996). Las aguas subterráneas de Costa Rica:Un recurso en peligro. En Reynolds Vargas, J., (Ed.), Utilización y Manejo Sostenible de los Recursos Hídricos. (pp. 157-165). Heredia, Costa Rica: Editorial Fundación UNA. http://www.una.ac.cr/hidrologia/

Reynolds-Vargas, J., Richter, D.D. y Bomemisza, E. (1994). Environmental impacts of nitrificationand nitrate adsorption in fertilized Andisols in the Valle Central of Costa Rica. Soil Science 157: 289-299. http://www.una.ac.cr/hidrologia/

Reynolds, J. (2005). Contaminación de aguas subterráneas por compuestos orgánicos volátiles. Recuperado el 31 de $\quad$ enero $\quad$ del $\quad 2010$ en http://www.una.ac.cr/hidrologia/documentos/compuesto_organicos_volatiles.pdf (15/o9/2009)

Reynolds-Vargas, J., Araguas, L., Fraile, J., Castro, L. y Rozanski, K. (1995). Nitrate leaching through volcanic soils in the Central Valley, Costa Rica. En: Nuclear Techniques in Soil-Plant Studies for Sustainable Agriculture and Environmental Preservation. Proceedings of a Symposium. pp. 549- 559. Vienna, Austria, 17-21 October 1994. STI/PUB/947. http://www.una.ac.cr/hidrologia/

Rosero, L. (2003). Situación demográfica general de Costa Rica. Recuperado el 20 de noviembre del 2009 en http://ccp.ucr.ac.cr/bvp/pdf/cambiodemografico/Rosero-demografica-2003.pdf

Ruiz, O. (2009). Tribunal ambiental cerraría Dos Pinos por contaminación. Recuperado el 21 de setiembre del 2009

http://centralcostarica.net/index.php/noticias/nacionales relevantes/tribunal ambiental cerraria do s pinos por contaminacion .html

Sagot, A. (2007). Construcciones en cauces y áreas de protección. Ambientico 164: 16-17. Recuperado el 24 de octubre del 2009 en http://www.ambientico.una.ac.cr/164.pdf

Sánchez, A. (2007). Acciones sociales e inundaciones en el medio urbano. Ambientico 169: 8-9. Recuperado el 24 de octubre del 2009 en http://www.ambientico.una.ac.cr/169.pdf

Solano, V. y Bravo, J. (2007). Contaminación de acuíferos por contaminación de hidrocarburos en Costa Rica. Ambientito 171:12-14. Recuperado el 24 de octubre del 2009 en http://www.ambientico.una.ac.cr/171.pdf

Solano, J., Retana, J.A. y Villalobos, R. (2002). Inundaciones. Top. Meteoro. Oceanog. 9(2): 112-122.

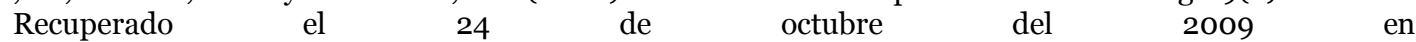
http://www.imn.ac.cr/publicaciones/revista/Solano\%20final\%20ultimo1202.pdf

UNEP.NET. (2006). Agua dulce. Recuperado el 11 de octubre del 2009 en http://www.centrogeo.org.mx/unep/documentos/CostaRica/CRagua.pdf 
Valdez, J. (2002). Gestión de la calidad del aire. Octavo informe sobre el estado de la nación en desarrollo humano sostenible. Recuperado el 11 de octubre del 2009 en http://www.estadonacion.or.cr/info2002/nacion8/Poarmonia/Gestion\%20de\%2ola\%20calidad\%20del\%20aire.pdf

Villegas, J. (2005). Lluvia ácida daña edificios y monumentos capitalinos. La Nación 20 de mayo de 2006. Recuperado el 11 de octubre del 2009 en http://www.nacion.com/ln ee/2006/mayo/20/pais8.html

\section{Nota acerca de la autora}

\section{Zaidett Barrientos Llosa}

Máster en Biología. Es investigadora y coordinadora de la Maestría Académica en Manejo de Recursos Naturales y del Laboratorio de Ecología Urbana, en la Universidad Estatal a Distancia (UNED).

Correo electrónico: zbarrientos@uned.ac.cr

\section{AGRADECIMIENTOS}

Se agradece el trabajo asistencial de Maribel Zúñiga Este trabajo es parte del trabajo doctoral de la autora dentro del Doctorado en Ciencias Naturales para el Desarrollo (DOCINADE) programa conjunto de la Universidad Nacional (UNA), Costa Rica, Instituto Tecnológico de Costa Rica (TEC) y la Universidad Estatal a Distancia (UNED), Costa Rica. La autora esta matriculada en la UNED. 